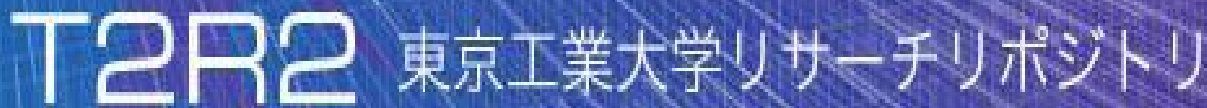

\section{Tokyo Tech Research Repository}

\section{論文 /著書情報 \\ Article /Book Information}

\begin{tabular}{|l|l|}
\hline Title & $\begin{array}{l}\text { Self-seeding Crystallization of Silicon Thin Films U sing Continuous- } \\
\text { Wave Laser }\end{array}$ \\
\hline Authors & Shin-Ichiro Kuroki, Shuntaro Fujii, Koji Kotani, Takashi Ito \\
\hline Citation & ECS Transactions, Vol. 2, No. 10, pp. 71-75 \\
\hline 発行日 /Pub. date & 2007,10 \\
\hline DOI & $\begin{array}{l}\text { http://dx.doi.org/10.1149/1.2408945 } \\
\text { 権利情報 /Copyright }\end{array}$ \\
$\begin{array}{l}\text { (c) The Electrochemical Society, Inc. 2007. All rights reserved. Except } \\
\text { as provided under U.S. copyright law, this work may not be reproduced, } \\
\text { resold, distributed, or modified without the express permission of The } \\
\text { Electrochemical Society (ECS). The archival version of this work was } \\
\text { published in ECS Transactions, Vol. 2, No. 10, pp. 71-75 }\end{array}$ \\
\hline
\end{tabular}




\title{
Self-seeding Crystallization of Silicon Thin Films Using Continuous-Wave Laser
}

\author{
Shin-Ichiro Kuroki, Shuntaro Fujii, Koji Kotani, and Takashi Ito \\ Graduate School of Engineering, Tohoku University \\ 6-6-05 Aza-Aoba, Aramaki, Aoba-ku, Sendai, 980-8579 Japan
}

\begin{abstract}
Low temperature crystallization of amorphous silicon thin films using continuous-wave laser was investigated. An overlapping of laser beam exposure enhanced the crystallization of a polycrystalline Si thin film, and the silicon crystal nucleus grew its size laterally. A laser beam spot had an elliptical shape for a gradual slope of temperature in a laser irradiated region, and was elongated to a scanning direction. The elongated laser spot with a gradual slope of temperature made a crystallization time longer. Consequently, a two dimensional lateral crystallization of (100) well-oriented Si thin films was achieved.
\end{abstract}

\section{Introduction}

Recrystallization of silicon thin films has been investigated for application to thin film transistors (TFTs). Currently, low-temperature polycrystalline-silicon (LTPS) TFTs has been used in active-matrix liquid crystal display technologies. Well-crystallized silicon thin films that have large grains are needed to make high performance TFTs for new emerging applications, such as system LCD and paper-like display. Various crystallization methods such as the eximer-laser annealing (ELA), the phase-modulated ELA (PMELA), the sequential lateral solidification (SLS), the metal-induced lateral crystallization (MILC), and the continuous-wave laser lateral crystallization (CLC) have been proposed to improve the TFT performances [1-4]. The CLC technology has attracted attention due to its controllability and high field effect mobility. By using a diode-pumped solid state (DPSS) continuous wave (CW) laser, an amorphous Si thin films are crystallized continuously, the CLC technology achieved one dimensional long and narrow grain [4],

The Purpose of this work is to form two dimensional large grains of Si thin films using DPSS CW laser. In this paper, effects of laser spot shape and overlap of laser irradiated region on the recrystallization of thin a-Si films were investigated

\section{Experiments}

Figure 1 shows a schematic of the sample used for the laser crystallization experiments. An amorphous silicon (a-Si) thin film was deposited by LPCVD method using $\mathrm{SiH}_{4}$ on a plasma-CVD $\mathrm{SiO}_{2}(800 \mathrm{~nm}) /$ thermal $\mathrm{SiO}_{2}(100 \mathrm{~nm}) / \mathrm{Si}(100)$ substrate. The thickness of the a-Si film was $150 \mathrm{~nm}$. After the a-Si film deposition, a cap $\mathrm{SiO}_{2}$ film was deposited by plasma-CVD. The purpose of the cap $\mathrm{SiO}_{2}$ deposition is anti-reflection for laser irradiation. The DPSS-CW laser with the wavelength of $\lambda=532 \mathrm{~nm}$ was used for silicon melting. The laser beam was guided by laser optics to reach a sample wafer. The sample wafer was placed on an X-Y stage. This stage was moved along two directions, 


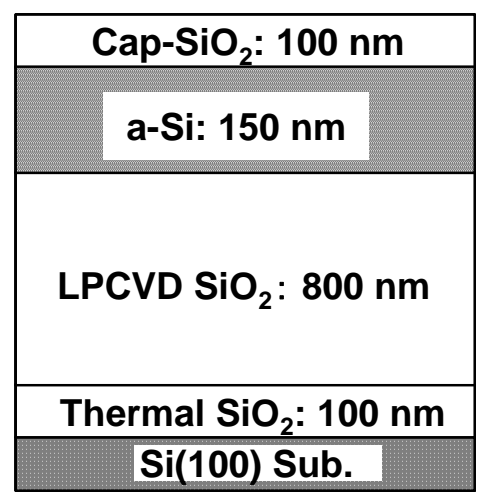

Fig.1 Schematic diagram of sample structure used in the laser recrystallization experiments.

and a laser beam scanned over sample wafer. Laser power was varied from 5.0 to $10.0 \mathrm{~W}$. Scanning speed was also varied from 1 to $100 \mathrm{~cm} / \mathrm{s}$. The width of $\mathrm{Si}$ melted region at $7.0 \mathrm{~W}$ laser power and $10 \mathrm{~cm} / \mathrm{s}$ speed was $48 \mu \mathrm{m}$. The overlap region of laser irradiation was varied from overlap ratio 0 to $90 \%$, and orientation of Si films was investigated by X-Ray Diffraction (XRD).

\section{Overlap effect on silicon crystallization}

The optical constants for $\mathrm{Si}$ thin films were measured by spectroscopic ellipsometry with TaucLorentz calculation. A refractive index of the a-Si thin film was 4.70. After the laser annealing, the refractive index decreased to 4.139. A conventional poly-Si film deposited by LPCVD and a conventional Si single crystal had refractive index 4.189 and 4.134, respectively and then the refractive index after laser annealing was smaller than that of a conventional poly-Si film. Optical adsorption coefficient at $\lambda=532 \mathrm{~nm}$ was also measured. Adsorption coefficient (imaginary part of complex refractive index) of the a-Si film was 0.574, and the a-Si film has high optical adsorption for $532 \mathrm{~nm}$, which means the a-Si film melts easily by the $532 \mathrm{~nm} \mathrm{CW}$ laser. On the other hand, after the laser annealing, adsorption coefficient of a re-crystallized Si film was zero, therefore a re-crystallized Si film was transparent to $532 \mathrm{~nm}$ laser light.

Figure 2 shows the result of the out-of-plane XRD measurement. Before a laser annealing, the a-Si film had no orientation of silicon crystal. After the laser annealing, orientations of Si crystal were observed. The diffraction peaks of Si Miller indices (111), (220) and (311) appeared in 2theta angle $28.48^{\circ}, 47.40^{\circ}$ and $56.28^{\circ}$, respectively. The peak intensity depended on the overlap ratio. From the overlap ratio 0 to $40 \%$, the (220) orientation increased. This means that crystallization of the Si films grew. Above $40 \%$ overlap ratio, the (220) orientation decreased, and (111) orientation increased. The crystallization of Si thin film was developed, and the overlap effect of Si crystallization on (111)/ (220) diffraction intensity ratio is shown in Fig. 3.

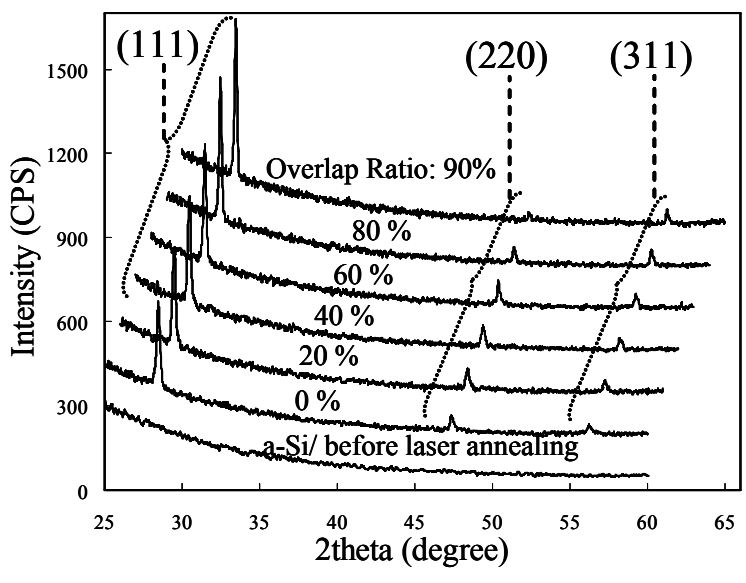

Fig. 2. Out-of-plane X-ray diffraction curves of silicon thin films. Overlap ratio was varied from 0 to $90 \%$.

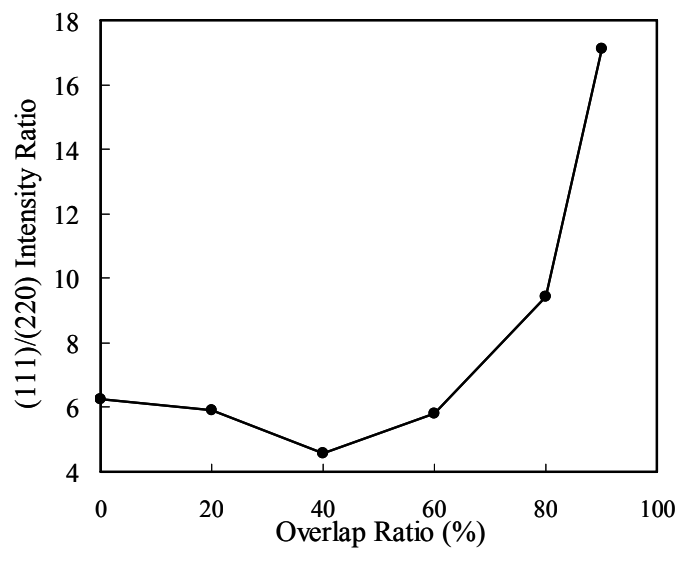

Fig. 3. (111)/(220) diffraction intensity ratio as a function of overlap ratio. This intensity ratio was calculated from the out-of-plane XRD profile. 

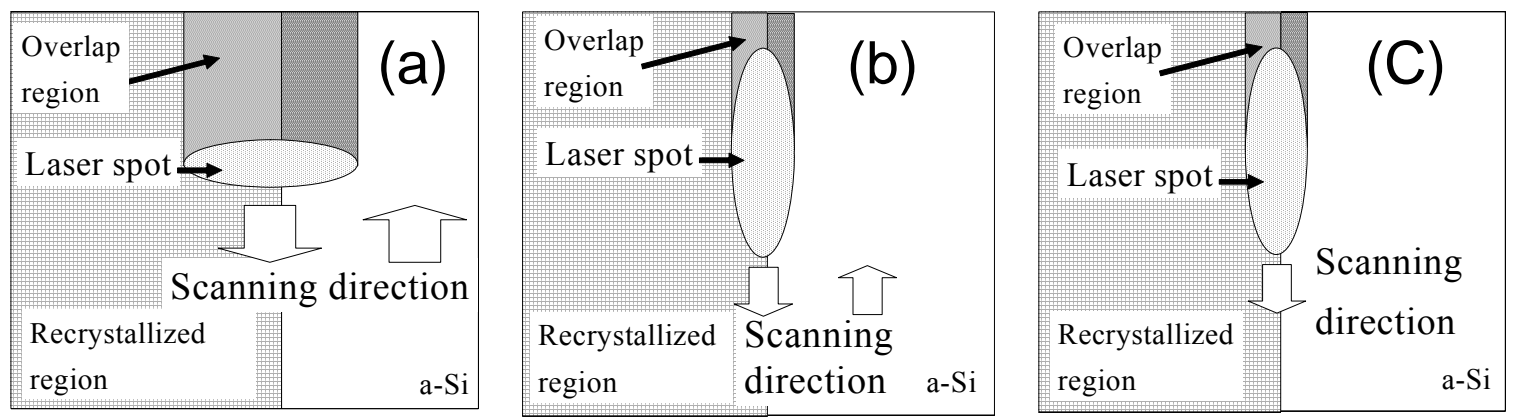

Fig 4. Schematic diagrams of laser beam shapes and scanning methods: (a). a conventional spot shape with zigzag scanning, (b). an elongated spot shape with zigzag scanning, (c). an elongated spot shape with raster scanning.

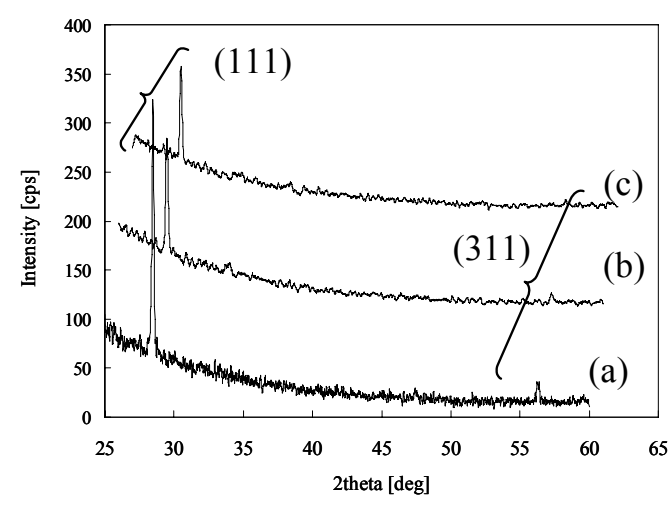

Fig 5. The out-of- plane XRD spectra: (a). a conventional spot shape with zigzag scanning, (b). an elongated spot shape to a scanning direction with zigzag scanning, (c). an elongated spot shape to a scanning direction with raster scanning.

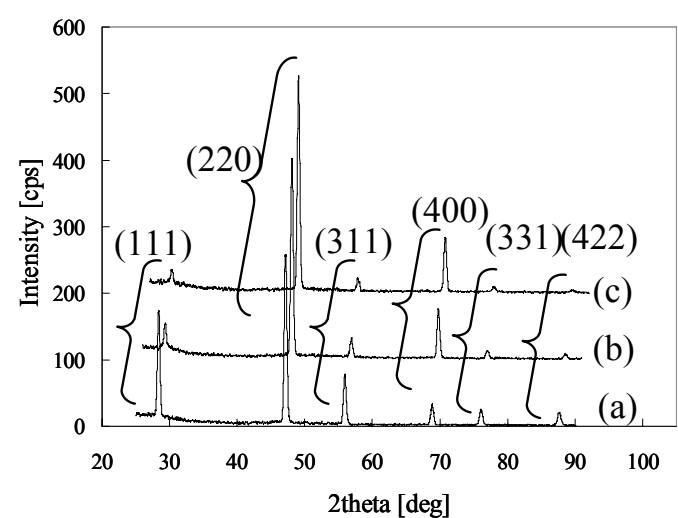

Fig 6. The in-plane XRD spectra: (a). a conventional spot shape with zigzag scanning, (b). an elongated spot shape to a scanning direction with zigzag scanning, (c). an elongated spot shape to a scanning direction with raster scanning.

\section{Laser spot shapes and scanning methods}

Figures 4 shows schematic diagrams of laser spot shapes and the scanning methods. Figure 4 (a) shows a conventional one, that had an elliptical beam shape and scanned a

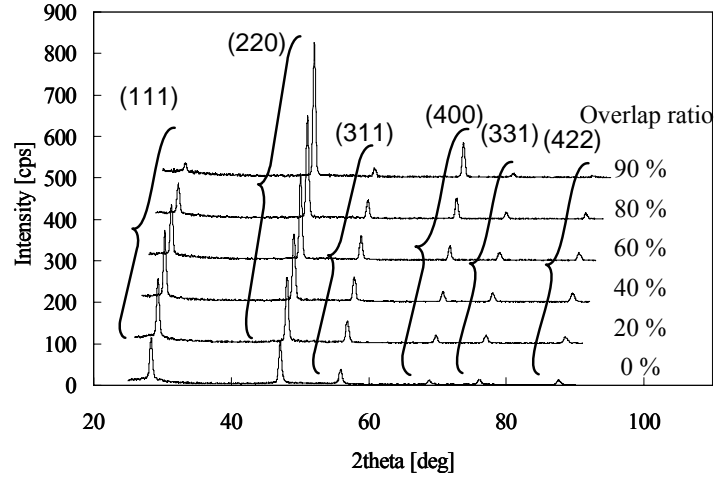

Figure 7. In-plane XRD spectra of dependency on overlap ratio. Laser annealing condition is a combination of an elongated spot shape and one-way scanning. sample wafer to the direction of minor axis. In Fig.4 (b) and (c), the laser spot had also an elliptical shape and scanned a sample wafer to the direction of the major axis with a zigzag scanning and a raster scanning, respectively. Figure 5 shows out-ofplane XRD spectra of recrystallized $\mathrm{Si}$ thin films. By changing a laser spot shape from a conventional one to an elongated one, all peaks in the out-ofplane spectra were decreased. Figure 6 shows in-plane XRD spectra of 

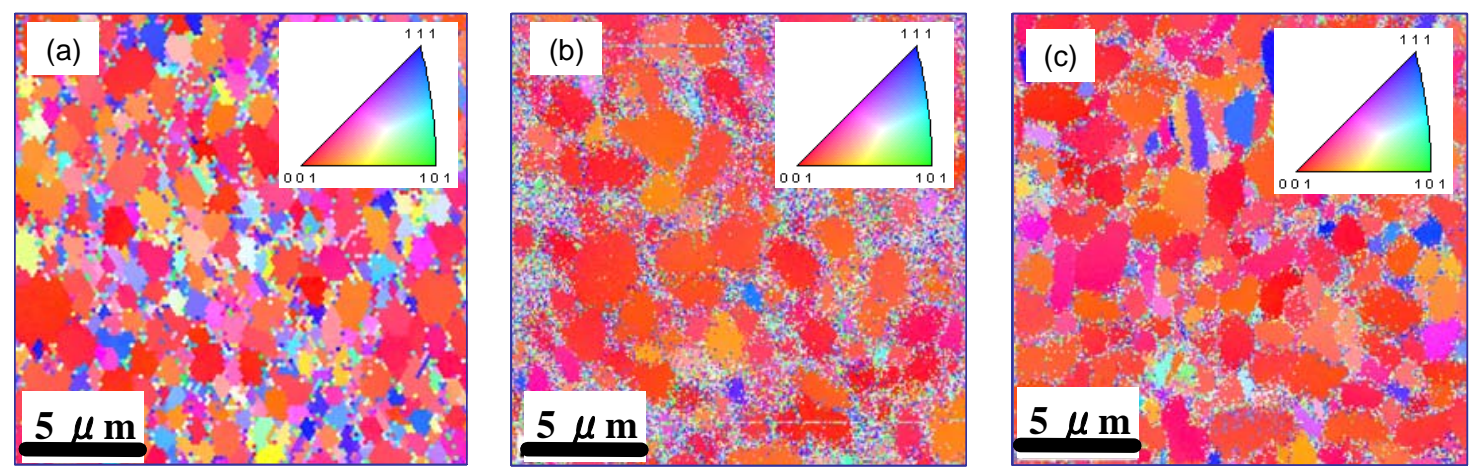

Fig 8. EBSP patterns: (a). a conventional spot shape with zigzag scanning, (b). an elongated spot shape with zigzag scanning, (c). an elongated spot shape with raster scanning.

recrystallized Si thin films. The (220) and (400) peaks were drastically increased, and other peaks in in-plane spectra were greatly decreased. In addition, by changing laser scanning methods from a zigzag scanning to a raster scanning, all peaks in out-of-plane spectra were more decreased, and (220) and (400) peaks in in-plane spectra increased more and other peaks in in-plane spectra decreased also. It is found that a laser recrystallization with an elongated laser spot and a raster scanning effectively increased crystallization of thin Si films. Figure 7 shows in-plane XRD spectra of recrystallized thin Si films as a function of overlap ratio. The overlap ratio was changed from $0 \%$ to $90 \%$. A laser recrystallization was carried out with an elongated laser spot and a raster scanning. As overlap ratio was increased, (220) and (400) peaks increased, and other peaks decreased. This means that crystal-grains of the Si thin films were enhanced.

Figure 8 shows the electron back scattering pattern (EBSP) mappings. Figures 8 (a), (b), and (c) are the results of laser recrystallization by a conventional spot with a zigzag scanning, an elongated spot with a zigzag scanning, and an elongated spot with a raster scanning, respectively. By changing a laser spot from a conventional spot to an elongated spot, the (100) area was increased. And by changing a laser scanning method from zigzag scanning to raster scanning, the grain boundaries became clear, and also the (100) area was increased. Figure 9 shows grain size distributions calculated from EBSP mappings.

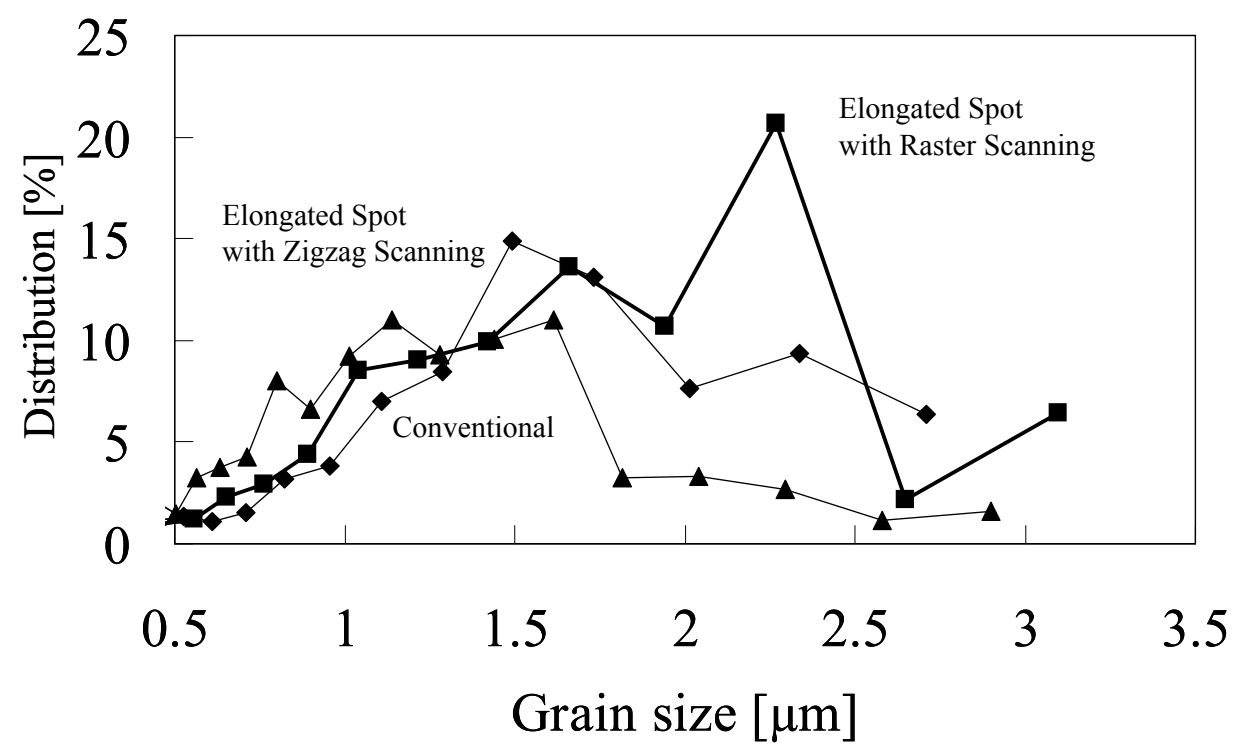

Fig 9. Grain size distributions calculated from the EBSP patterns. 
The average grain sizes were 1.16, 1.32, and $1.60 \mu \mathrm{m}$ for the conventional laser spot with zigzag scanning, the elongated spot with zigzag scanning, and the elongated laser spot with raster scanning, respectively. By using an elongated spot with a raster scanning, a (100) well-oriented thin Si film was obtained and average grain size of $1.60 \mu \mathrm{m}$ was achieved.

\section{Conclusion}

Low temperature crystallization of amorphous silicon (a-Si) thin films using a diodepumped solid state (DPSS) continuous wave (CW) laser was investigated. A laser beam spot was designed to be elongated to a scanning direction in order to form a gradual slope of temperature in a laser irradiated region. The elongated-laser spot with a raster scanning successfully enhanced a crystallization of Si thin films. Consequently, a large (100) welloriented crystal-grains of Si thin films have been obtained.

\section{Acknowledgment}

A part of this work was supported by Special Coordination Fund for Promoting Science and Technology of Ministry of Education, Culture, Sports, Science and Technology (MEXT).

\section{References}

1. M. Matsumura, AM-LCD 05, p. 1-4 (2005).

2. Y.-J. Kim, K-S. Hwang, Y-I. Park, S-W. Lee, H. Kang, K. M. Lim, C-D Kim, and I-J. Chung, AM-LCD 05, p. 249-252(2005).

3. N. Higashi, G. Nakagawa, T. Asano, Mi. Miyasaka, and J. Stoemenos, AM-LCD 05, p. 289-292 (2005).

4. A. Hara, M. Takei, F. Takeuchi, K. Suga, K. Yoshino, M. Chida, T. Kakehi, Y. Ebiko, Y. Sano, and N. Sasaki, Jpn. J. Appl. Phys., 43(4A), p.1269 (2004). 